\title{
Caracterización biocinemática, al paso guiado a la mano, del caballo fino chilote
}

\author{
Biokinematic characteristics of the chilote horse hand led walk
}

\author{
A Escobar*, T Tadich \\ Instituto de Ciencia Animal y Tecnología de Carnes, Facultad de Ciencias Veterinarias,
} Universidad Austral de Chile, Casilla 567, Valdivia, Chile.

\begin{abstract}
SUMMARY
In the present study a description of some basic conformational and biokinematic characteristics of 16 mares that belong to the breed Caballo Fino Chilote was conduce, at the hand-led walk. The objective was to determine some conformational and biokinematic characteristics at the hand led walk for 16 mares and the possible aptitudes, of this breed, for being used as a therapeutic tool in hipotherapy activities. The conformational parameters were measured in vivo using a metric tape and ruler. For the time-related and biokinematic parameters, a $10 \mathrm{~m}$ curtain graduated every $50 \mathrm{~cm}$ was specially made, in front of which each mare was guided at a hand-led walk while being filmed with a Sony Hi-8 camera. The video was transformed into photos using the Final Cut programme; 15 frames per second were obtained in JPG format; the frames were then passed into TIFF to measure the angular parameters with the help of the Scion Image programme. Lineal parameters were directly measured from the video using a Sony flat screen television. All data analysis was conducted with descriptive statistics using Microsoft Excel xp. The means obtained for conformational parameters were: height at the withers $117.94 \mathrm{~cm}( \pm 2.6)$, height at the back $113.3 \mathrm{~cm}( \pm 2.1)$, and height at the hindquarters $119.9 \mathrm{~cm}( \pm 3.1)$. For the lineal parameters the mean stride length was $135.3 \mathrm{~cm}( \pm 8.9)$ and the mean step length was $69.3 \mathrm{~cm}( \pm 5.8)$. The mean stride duration was 1.18 seconds giving a mean speed of $114.7 \mathrm{~cm} / \mathrm{sec}$. The angular parameters of the limbs were measured for the shoulder, elbow and carpus joints in the forelimb and for the hip, stifle and tarsus joints in the hindlimb, also the scapula and pelvis inclination were measured obtaining curves for each one along a complete stride cycle, and angular range of motion was determined.
\end{abstract}

Palabras clave: caballo chilote, biocinemática, videografía, hipoterapia.

Key words: Chilote horse, biokinematics, videography, hipotherapy.

\section{INTRODUCCION}

En los animales domésticos, la raza corresponde a un grupo de individuos que, como consecuencia de los métodos de reproducción y de selección practicados durante generaciones, poseen algunas características perceptibles en común, que posteriormente se transmitirán a las generaciones sucesivas (Briggs 1969). En Chile, la raza más popular de equinos es la raza "Caballo Chileno", de la cual existen registros de pedigrí que la describen detalladamente desde el año 1893 (Pinochet 1980). Existe además otro grupo más reducido de caballares relacionado con la Isla de Chiloé, el Mampato o Caballo Fino Chilote (Cabrera 1945).

El Caballo Fino Chilote es un recurso equino único en el mundo; antecedentes históricos, características morfológicas (Voeltz 1996) y un análisis genético (Barrera 1998) confirman que tiene su origen en aquellos

Aceptado 05.07.2005

*aescobar@uach.c ejemplares equinos provenientes de la península ibérica (FIA 2003*). Desde 1999 existen registros genealógicos para la raza Caballo Fino Chilote y existen dos vías para que los animales pasen a integrarlo, la genealógica y por calificación (FIA 2003*).

La conformación física del equino es el resultado de la evolución natural y las demandas ambientales, las cuales han determinado una evolución gradual de las propiedades locomotoras. A ello se le suma que el hombre ha criado caballos con el fin de obtener distintas fuerzas, velocidad o belleza de acuerdo a sus necesidades, llegándose a obtener diferencias considerables entre razas (Cano y col 2001). Los patrones de movimiento característicos de cada raza son el resultado de una selección según la función requerida por el hombre y las necesidades impuestas por la época como es el caso de los caballos para batallas, para silla, deporte o trabajo (Galisteo y

* FIA. 2002. (Fundación para la Innovación Agraria). Proyecto: Recuperación y multiplicación del caballo chilote. Disponible en http://www.fia.cl/difus/proejem/ejem40.htm. Consultado el 3 de mayo del 2003 
col 1997). Existen, entonces, diferencias en la conformación por las cuales algunos caballos se adaptan mejor que otros a determinados trabajos; esas diferencias que caracterizan a los caballos de silla, de carrera, los de tiro pesado, de cacería, etc. (Ensminger 1973).

Uno de los parámetros que se utiliza para describir y clasificar caballares, es el movimiento. Badoux (1994) define la biocinemática como el análisis de los movimientos sin tener en cuenta las fuerzas que lo producen; un ejemplo de esto es el análisis cinematográfico de los movimientos de los miembros del caballo durante el paseo. Partiendo de la base de que los caballos cuando son examinados en condiciones similares tienden a repetir su patrón de movimiento, el método de análisis de la locomoción normal se puede estandarizar, y aplicando métodos de análisis apropiados se pueden determinar diferencias en el comportamiento locomotor de animales de diferentes características, como la raza (Galisteo y col 1999).

La evaluación de los movimientos del caballo es corrientemente realizada de manera visual por jueces, método que no sólo depende de la subjetividad del criterio de los jueces sino también de las limitaciones del ojo humano (Morales y col 1998). Sin embargo, se han desarrollado técnicas más objetivas, como la videografía de velocidad normal que, a pesar de ser menos precisa que la videografía de alta velocidad, tiene ventajas como su menor costo y mayor versatilidad (Morales y col 1998). Otras técnicas objetivas son la histoquímica muscular y pruebas fisiológicas, que permiten cuantificar algunas características funcionales del caballo (Galisteo y col 1999). Según Morales y col (1998), existe también un incremento en el uso de parámetros angulares de batida para la descripción de la locomoción equina con bases cuantitativas, ya que entrega información inmediata sobre las causas determinantes de los parámetros de batida.

Conocer el patrón de movimiento de un caballo es esencial para determinar los posibles usos que se le pueden dar; este es el caso para determinar si son aptos para la hípica, equitación, adiestramiento y especialmente para la posible utilización de un caballo para hipoterapia. El objetivo de este estudio fue describir algunas variables conformacionales y biocinemáticas básicas, al paso guiado a mano, en yeguas de la raza Caballo Fino Chilote y relacionarlas con las posibles aptitudes de esta raza para ser utilizada como herramienta terapéutica en la actividad de hipoterapia.

\section{MATERIAL Y METODOS}

Se utilizaron 16 hembras adultas de la raza Caballo Fino Chilote, mayor de tres años, pertenecientes al Fundo Teja Norte de la Universidad Austral de Chile, Valdivia, y a un criadero de Caballos Fino Chilote ubicado en Puerto Montt, Chile. La medición de los parámetros de movimiento se llevó a cabo de la siguiente forma:

Medición de las variables conformacionales. Con la ayuda de un bastón hipométrico se obtuvo el valor en centímetros de la Alzada a la Cruz, hasta la porción más alta de la cruz; la Alzada a la Espalda, hasta la sección media de la espalda; y la Alzada a la Grupa, hasta el punto más alto de la grupa. Todas desde el suelo en forma perpendicular.

Medición de las variables lineales. Se realizó ubicando al caballo en un plano horizontal de $10 \mathrm{~m}$ en un potrero de pasto; luego se le hizo caminar al paso, guiado a mano, frente a un telón de 1,6x10 m graduado cada $50 \mathrm{~cm}$, a lo largo de los $10 \mathrm{~m}$ y a una distancia de $12 \mathrm{~m}$ de la ubicación de la cámara. Con una cámara de video Sony Hi-8 se registraron dos pasadas para cada caballo. Las imágenes de cada pasada se observaron en un televisor Sony pantalla plana de $25^{\prime}$, desde el cual se obtuvieron cuatro datos para cada una de las siguientes variables.

Largo del Paso. Se midió con cinta métrica $(\mathrm{cm})$ la distancia entre las huellas de los dos miembros delanteros (Adams 1985), o la distancia entre el posicionamiento de cascos contralaterales (AD-AI) (Clayton 1995).

Largo de la Batida. Se midió, con cinta métrica (cm), la distancia entre dos contactos sucesivos del mismo casco (AD), resultando por lo tanto más larga que un paso (Adams 1985).

Medición de Velocidad. Se determinó el promedio de velocidad de batida, para cada individuo y para el grupo, la cual fue calculada como el largo de la batida en centímetros dividido por la duración de la batida, en segundos (Clayton 1995). Para esto se utilizó un cronómetro obteniendo 4 datos para cada animal, los cuales fueron promediados.

Medición de los cambios angulares de los miembros. Se marcaron puntos anatómicos con cinta adhesiva para el miembro anterior (figura 1) y posterior (figura 2). Luego se ubicó al caballo en un plano horizontal, utilizando el telón graduado y se hizo caminar al paso guiado a mano por delante del mismo, registrándose para cada caballo dos pasadas con la cámara de video (figura 4).

Las yeguas utilizadas no presentaban ningún signo de afección evidente del aparato locomotor al momento de realizar las mediciones.

El video se traspasó a formato JPG con el programa Final Cut Pro V.1.2.5 para iMac, obteniendo para cada ciclo (correspondiente a una batida) 15 fotografías por segundo, lo cual correspondió a un total de 16 a 25 fotografías por ciclo. Con ayuda del programa Adobe Photoshop (Copyright (c) 1989-2000 Adobe Systems 

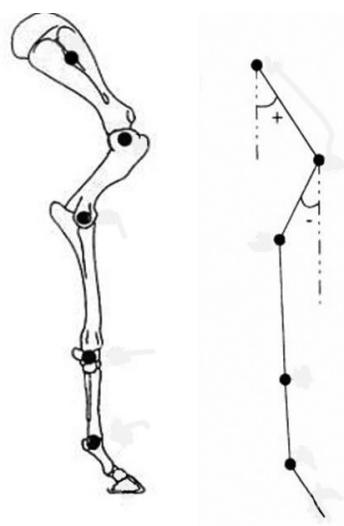

Figura 1. Ubicación de marcas en el miembro anterior, de proximal a distal: Tuberosidad de la espina de la escápula, parte lateral del tubérculo mayor del húmero, epicóndilo lateral del húmero, Carpal ulnar, Area metacarpal de inserción del ligamento colateral de la articulación del nudillo.

Location of markers, for the forelimb, in a proximal to distal sequence: The tuber spinae scapulae, the lateral part of the greater tubercle of the humerus; the lateral epicondyle of the humerus; the ulnar carpal bone; the metacarpal attachment of the lateral collateral ligament of the felock joint.

Inc.) se ajustó el brillo y contraste a cada fotografía y se traspasaron a formato Tiff. Una vez obtenidas las 586 fotografías en formato Tiff se utilizó el programa Scion Image (Copyright 1997-2000, Scion Corporation) para la medición de los cambios angulares de los miembros. Los resultados se presentaron en curvas que se normalizaron, para el total de individuos, al promedio de duración de la batida (100\% batida)

Medición del ángulo de inclinación pélvica (A.I.P). En cada una de las fotografías (JPG) se utilizó el programa Adobe Photoshop 6.0 para marcar una línea horizontal que pasaba por la marca correspondiente al área ventral de la tuberosidad coxal, para luego formar un ángulo con la marca correspondiente al trocánter mayor del fémur (figura 3 izquierda). Estas fotografías se pasaron a formato Tiff para medir la amplitud del ángulo, en grados, a través del programa Scion image. Para cada fotografía se obtuvo un grado de inclinación. La amplitud del movimiento se obtuvo de la resta entre el mayor grado y el menor grado del A.I.P.

Medición del ángulo de inclinación de la escápula (A.I.E). Se marcó una línea imaginaria paralela al plano horizontal que pasaba por la marca correspondiente a la tuberosidad de la espina de la escápula, para luego formar un ángulo con la marca correspondiente a la porción lateral de la tuberosidad del húmero (figura 3 derecha). Luego se aplicó la misma metodología utilizada para la medición del A.I.P.

Análisis de los resultados: Se confeccionaron tablas para las variables lineales de alzada (cruz, grupa y espalda),

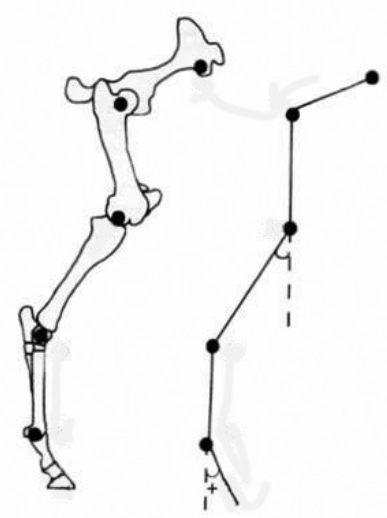

Figura 2. Ubicación de marcas en el miembro posterior, de proximal a distal: Area ventral de la tuberosidad coxal, Trocánter mayor del fémur, Cóndilo femoral, en el lugar de inserción del ligamento colateral lateral, Talo, Area metatarsal de inserción del ligamento colateral lateral de la articulación del nudillo.

Location of markers for the hindlimb, in a proximal to distal sequence: ventral part of the tuber coxae; greater trochanter; femoral condyle at the attachment of the lateral collateral ligament; talus; metatarsal attachment of the lateral collateral ligament of the fetlock joint.

largo de batida, largo del paso y velocidad; se utilizó la herramienta de estadística descriptiva del programa Microsoft Excel de Microsoft Office xp. Para calcular media, coeficiente de variación, rango, desviación estándar, coeficiente de correlación múltiple y coeficiente de determinación $\mathrm{R}^{2}$. Para el análisis y confección de tablas y gráficos de las variables temporales (cambios angulares), del miembro posterior y anterior derecho, se utilizó el mismo programa, calculándose porcentajes, media, rango y curva de tendencia (Polinomial de orden 6).

\section{RESULTADOS}

Variables conformacionales. El cuadro 1 muestra que las 16 yeguas registradas presentaron una alzada a la cruz promedio de $117,9 \mathrm{~cm}$; promedio que no supera a la alzada a la grupa, mientras que la alzada a la espalda fue siempre menor.

Variables lineales. En el cuadro 2 se observa que el largo promedio de una batida, para las 16 yeguas muestreadas, fue de $135,3 \mathrm{~cm}( \pm 8,9 \mathrm{~cm})$, siendo la longitud mínima alcanzada de $113,2 \mathrm{~cm}$ y la máxima de $150,9 \mathrm{~cm}$ para una batida; para el largo del paso se obtuvo un promedio de $69,3 \mathrm{~cm}( \pm 5,8 \mathrm{~cm})$, teniendo el paso más corto una longitud de $56.6 \mathrm{~cm}$ y el más largo una longitud de $83,0 \mathrm{~cm}$.

Al calcular la relación entre el largo de la batida y el largo de un paso se pudo observar que un paso corresponde a aproximadamente un $50 \%$ de una batida de cada yegua. 

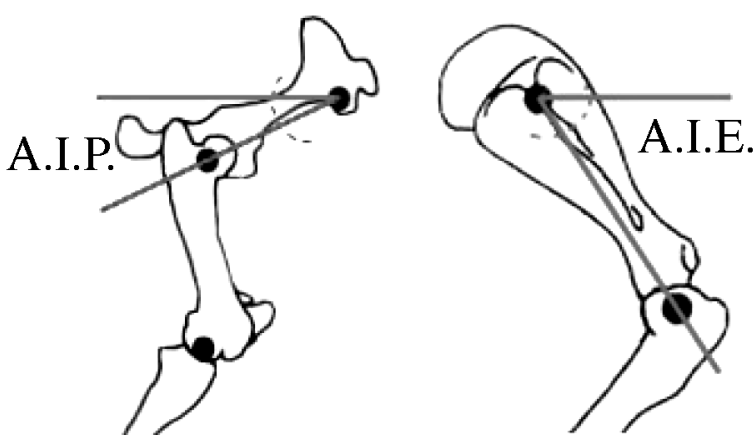

Figura 3. Izquierda; diagrama del ángulo de inclinación pélvica. Derecha; diagrama del ángulo de inclinación de la escápula.

Left; diagram of the pelvic inclination angle. Right; diagram of the scapulae inclination angle.

Cuadro 1. Media, mediana, moda, desviación estándar (DE) y rango de distribución de las alzadas a la cruz, espalda y grupa, en centímetros, para las 16 yeguas.

Mean, median, standard deviation and range of distribution for height of the withers, the back and the hindquarter for the 16 mares.

\begin{tabular}{lccc}
\hline VARIABLE & A. Cruz & A. Espalda & A. Grupa \\
\hline Media & 117,9 & 113,3 & 119,9 \\
Mediana & 118,5 & 113 & 119 \\
DE & 2,6 & 2,1 & 3,1 \\
Rango & $113-122$ & $110-118$ & $115-126$ \\
\hline
\end{tabular}

Cuadro 2. Media, mediana, desviación estándar (DE), coeficiente de variación (CV\%) y rango del largo de la batida y del paso para las 16 yeguas.

Mean, median, standard deviation, variation coefficient and range of the length of the stride and step for the 16 mares.

\begin{tabular}{lcc}
\hline VARIABLE & Largo batida & Largo paso \\
\hline Media & 135,3 & 69,3 \\
Mediana & 135,9 & 69,8 \\
DE & 8,9 & 5,8 \\
CV\% & 6,6 & 8,4 \\
Rango & $113,2-150,9$ & $56,6-83,0$ \\
\hline
\end{tabular}

Velocidad. La velocidad promedio que alcanzaron las 16 yeguas al paso guiado a la mano fue de $114,7 \mathrm{~cm} / \mathrm{seg}$ y el tiempo promedio que demoraron en realizar una batida completa, de $135,3 \mathrm{~cm}$, fue de 1.2 segundos.

Cambios angulares de los miembros. En el miembro anterior la articulación que presentó cambios angulares menores a través de una batida fue la articulación del hombro (figura 5), mientras que aquella que presentó mayores cambios fue la articulación del carpo (figura 7). Para las articulaciones del hombro, codo y carpo la máxima flexión se alcanzó entre el $60-80 \%$ de la batida (figuras 5,6 y 7$)$.

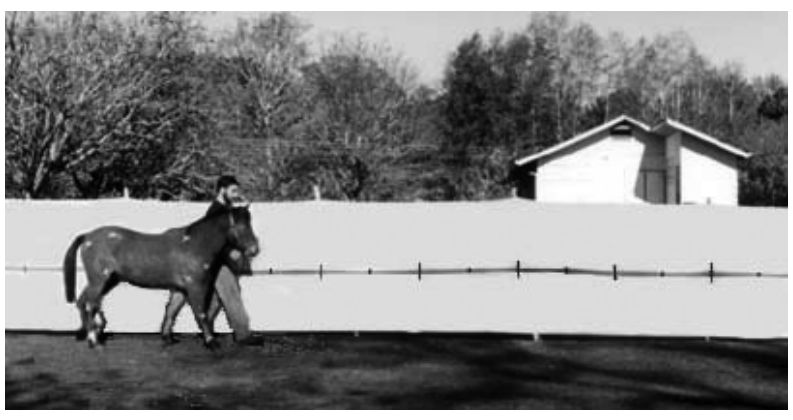

Figura 4. Yegua con las marcas sobre la piel y guiada al paso por delante del telón de $10 \mathrm{~m}$ de longitud.

Mare with the skin marks being guided at a hand led walk, in front of a $10 \mathrm{~m}$ curtain.

En el miembro posterior la articulación que presentó cambios angulares menores a través de una batida fue la articulación de la cadera (figura 8), mientras que aquella que presentó mayores cambios fue la articulación del corvejón (figura 10). Las articulaciones de la cadera y del corvejón alcanzaron la máxima flexión entre el 40$60 \%$ de la batida (figuras 8 y 10) mientras que la articulación de la rodilla lo hizo entre el $20-40 \%$ de la batida (figura 10).

En el cuadro 3 se aprecia que, de las articulaciones descritas, las que presentan una mayor amplitud o rango de movimiento son la articulación del carpo y la articulación del corvejón, siendo las mismas las que alcanzan un máximo grado de extensión. Aquellas que logran un grado de flexión mayor son la articulación del codo y la articulación de la cadera.

\section{DISCUSION}

La raza, sexo y edad tienen una influencia significativa sobre la conformación y variables biocinemáticas (Holmström 1990). Es por esto que en el presente estu-

Cuadro 3. Valores promedio de máxima extensión, máxima flexión y amplitud (en grados), para cada ángulo del miembro anterior y posterior derecho de las 16 yeguas.

Mean values of maximum extension, maximum flexion, and angular range of motion (degrees) for each angle in the forelimb and hindlimb for the 16 mares.

\begin{tabular}{lccc}
\hline ANGULO & Máx. Ext. & Máx. Flex. & Amplitud \\
\hline Inc. escápula & 72,9 & 53,8 & 19,2 \\
Art. hombro & 94,2 & 80,9 & 13,3 \\
Art. codo & 136,3 & 68,3 & 67,9 \\
Art. carpo & 175,4 & 105,6 & 69,8 \\
Inc. pelvis & 23,9 & 15,5 & 8,4 \\
Art. cadera & 81,4 & 63,9 & 17,4 \\
Art. rodilla & 131,3 & 85,8 & 45,5 \\
Art. corvejón & 156,9 & 105,9 & 50,9 \\
\hline
\end{tabular}



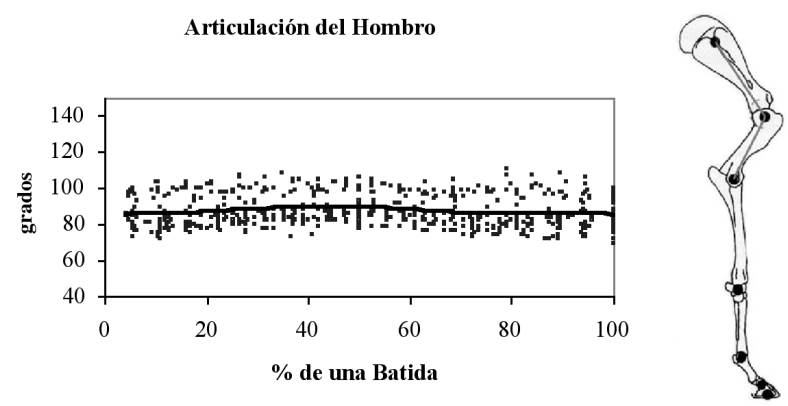

Figura 5. Curva de tendencia de las variaciones angulares (grados) para la articulación del hombro de las 16 yeguas, durante una batida completa.

Tendency curve of the angular variables (degrees) at the shoulder joint for the 16 mares during a complete stride.
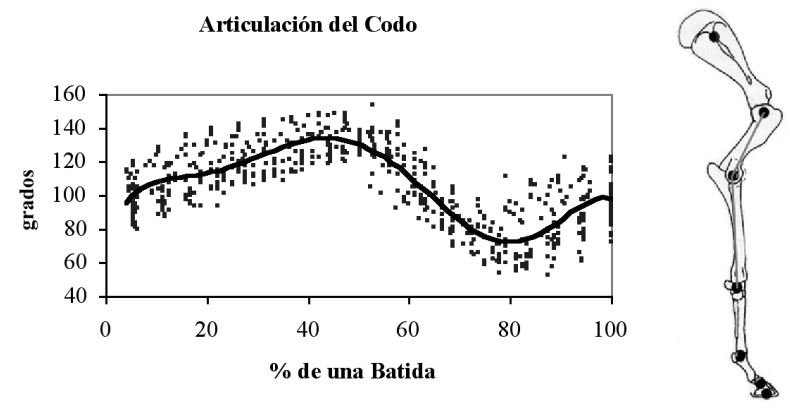

Figura 6. Curva de tendencia de las variaciones angulares (grados), para la articulación del codo, de las 16 yeguas, durante una batida completa.

Tendency curve of the angular variables (degrees) at the elbow joint for the 16 mares during a complete stride.

dio se utilizaron sólo hembras, mayores de tres años, de raza Caballo Fino Chilote, conformándose así un grupo homogéneo.

Dentro de las variables de conformación, la alzada a la cruz para el Caballo Fino Chilote ya había sido descrita por Cabrera (1945), observando que no superaba los $120 \mathrm{~cm}$. Luego en el estudio descriptivo de la morfología y distribución del Caballo Fino Chilote realizado por Voeltz (1996), se obtuvo una alzada a la cruz promedio de $119,9 \mathrm{~cm}$, encontrándose una diferencia entre machos y hembras, siendo los machos más altos; las hembras analizadas presentaron una alzada a la cruz promedio de 118,9 $\mathrm{cm}$. En el presente estudio, el valor promedio obtenido para la alzada a la cruz de las 16 yeguas estudiadas fue de 117,94 (cuadro 1), semejante al registrado por Voeltz (1996); además se pudo establecer las alzadas a la espalda y a la grupa promedio (cuadros 1 y 2), aspectos morfológicos que no se habían registrado en estudios anteriores para el Caballo Fino Chilote.

Es importante considerar la relación que existe entre las alzadas. $\mathrm{Al}$ respecto, la alzada a la cruz debiera ser tanto o más alta que la alzada a la grupa, ya que una cruz baja da lugar a molestias en el animal, además de no permitir mantener la montura en su lugar, y en casos extre-

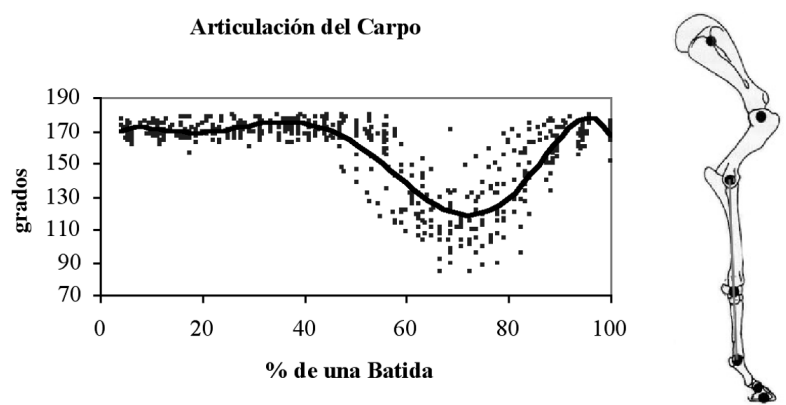

Figura 7. Curva de tendencia de las variaciones angulares (grados) para la articulación del carpo de las 16 yeguas, durante una batida completa.

Tendency curve of the angular variables (degrees) at the carpal joint for the 16 mares during a complete stride.

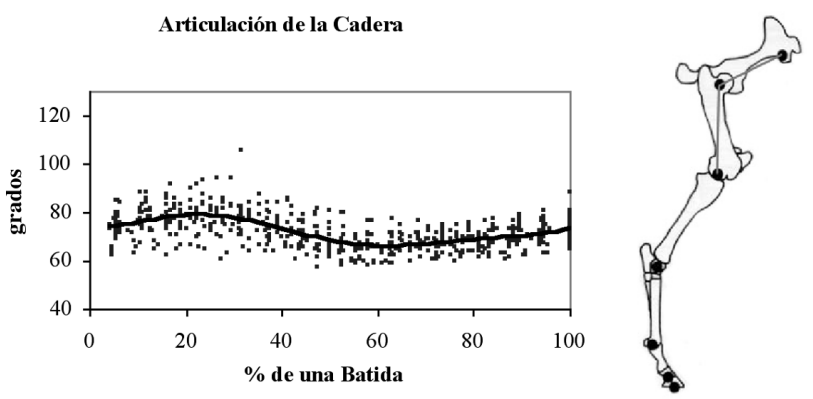

Figura 8. Curva de tendencia de las variaciones angulares (grados), para la articulación de la cadera de las 16 yeguas, durante una batida completa.

Tendency curve of the angular variables (degrees) at the hip joint for the 16 mares during a complete stride.

mos producir cierta incomodidad al jinete (Nordby 1971). En las yeguas estudiadas la alzada promedio a la grupa sólo superó en $2 \mathrm{~cm}$ a la alzada promedio a la cruz, siendo la moda igual para ambas alzadas $(119 \mathrm{~cm}$, cuadro 1$)$. La alzada del caballo ideal para hipoterapia no debiera ser mayor a $1,50 \mathrm{~cm}$, puesto que así se facilita la labor del fisioterapeuta (Soto 2002), teniendo por tanto el Caballo Fino Chilote una alzada óptima.

Una segunda variable de tipo lineal que se midió fue el largo de la batida que, como se definió anteriormente, corresponde a la distancia entre dos contactos sucesivos del mismo casco (Adams 1985). En este estudio se utilizaron los dos contactos sucesivos del miembro anterior derecho. Para la raza Caballo Fino Chilote no se encontraron antecedentes en la literatura revisa$\mathrm{da}$, siendo éste un estudio preliminar para la descripción de esta variable.

A pesar de que el paso es un aire que no requiere esfuerzo del caballo, existen diferencias entre caballos de distintas razas en los parámetros lineales de éste (Galisteo y col 1999). En cuanto a la longitud promedio de batida de 135,3 cm ( $\pm 8,9 \mathrm{~cm}$ de D.S) (cuadro 2), se observó una variación intraindividual y entre individuos similar a la obtenida por Galisteo y col (1999) para la 
raza Andaluz al paso guiado a mano (DS $\pm 10,2$ ) y a los resultados de Clayton (1995) para caballos de alto rendimiento al paso extendido ( $\mathrm{DS} \pm 9$ ).

Las diferencias conformacionales pueden influir en la variabilidad de los patrones cinemáticos. Dusek (1974) considera que la alzada a la cruz no está relacionada con la longitud de la batida en los diferentes aires, mientras que Galisteo y col (1998) mostraron que la alzada a la cruz está relacionada de manera positiva con los parámetros lineales al trote guiado a mano, y Galisteo y col (1999) destacan que los caballos que poseen una alzada a la cruz mayor son los que alcanzan una longitud de batida mayor, como es el caso del Anglo-Arabe.

Sin embargo, Cano y col (2001) encontraron que, en el caso de un estudio comparativo entre tres razas, Andaluces, Arabes y Anglo-Arabes, los Arabes a pesar de presentar la mayor alzada a la cruz fueron los que presentaron una longitud de batida ajustada menor. En este estudio se observó en forma similar que los individuos con una mayor alzada a la cruz no fueron los que alcanzaron una mayor longitud de batida, a pesar de existir una correlación positiva, pero extremadamente baja (coeficiente de correlación múltiple de 0,125).

Parte de la variabilidad de la batida, expresada por una alta desviación estándar entre e intraindividuos que se obtuvo en este estudio, podría estar dada por el hecho de que las yeguas fueron guiadas a mano y no se utilizó una trotadora como en otros estudios (Peham 2001). Además se debiera considerar la influencia del tipo de terreno en que se realizaron las mediciones (piso de tierra o pasto), el nulo entrenamiento y escaso manejo rutinario al que son sometidas las yeguas utilizadas en este estudio.

La variabilidad del largo de la batida es mayor en animales sanos que en aquellos que presentan algún grado de cojera, indicando esto una repetida aceleración y desaceleración en el caballo sano, que además sería la causa de un gasto de energía incrementado en éste (Peham 2001).

El largo promedio de la batida y del paso del Caballo Fino Chilote no sobrepasaron los datos obtenidos para las mismas variables en el humano por Lehmann (1999), por lo tanto ésta sería otra característica que hace al Caballo Fino Chilote apto para ser utilizado en hipoterapia.

La velocidad ha mostrado ser uno de los factores más importantes en la determinación de un patrón de locomoción equina (Cano y col 2001), destacándose que a velocidades iguales los caballos que poseen una mayor alzada son los que en general alcanzan una mayor longitud de batida (Galisteo y col 1999). La velocidad tendría una importante influencia en la mayoría de los parámetros de la batida, como también en los parámetros angulares de los miembros; este factor podría excluirse utilizando una trotadora (Buchner 2001). En el presente estudio la duración promedio de una batida al paso guiado a mano alcanzada fue de 1,2 segundos, correspondiendo a una velocidad promedio de $114,7 \mathrm{~cm} / \mathrm{seg}$.
En este estudio el caballo que logró una mayor longitud de batida, 146,2 cm, fue aquel que también alcanzó una mayor velocidad media de batida $(138,6 \mathrm{~cm} / \mathrm{seg})$ mientras que aquel que obtuvo una menor longitud media de batida $121,7 \mathrm{~cm}$ fue el que a su vez obtuvo una menor velocidad media de batida $(85,9 \mathrm{~cm} / \mathrm{seg})$. Lo anterior se asimila a los resultados obtenidos por Clayton (1995) donde la mayor velocidad alcanzada al paso medio y extendido se atribuyó a un incremento en el largo de la batida y disminución en la duración de ésta.

En cuanto a la variable paso, la longitud media alcanzada por las yeguas fue de $69,3 \mathrm{~cm}$ (cuadro 2), lo que correspondió al 51,23\% de la longitud promedio alcanzada para una batida $(135,3 \mathrm{~cm})$ y concuerda con los resultados obtenidos por Clayton (1995).

En el último tiempo existe un aumento en el uso de técnicas de análisis de los parámetros angulares de la batida para la descripción de la locomoción equina con bases cuantitativas, ya que entrega información de las causas inmediatas que determinan los parámetros de batida básicos (Morales y col 1998). En este caso se midieron para tres articulaciones del miembro anterior (articulaciones del hombro, codo y carpo) y tres del miembro posterior (articulaciones de la cadera, rodilla y corvejón), y se cuantificaron los cambios en la inclinación de la pelvis y la inclinación de la escápula, todo esto a través de una batida al paso guiado a mano. En cuanto a los valores obtenidos, no se encontraron referencias en la literatura para la raza Caballo Fino Chilote para ser comparadas, de manera que los resultados entregados representan un primer aporte.

Para la articulación del hombro, Kainer (1985) describe un ángulo de entre $120^{\circ}-130^{\circ}$ para el equino de pie, con un ángulo de $145^{\circ}$ en extensión y $80^{\circ}$ en flexión, sin mencionar a qué aire se realizaron las medidas o bajo qué condiciones. En las yeguas Chilotas se alcanzó una máxima extensión de $94,2^{\circ}$ y una máxima flexión de $80,9^{\circ}$ (cuadro 3), al paso guiado a mano. La variación que presentó la articulación del hombro durante el desarrollo de una batida completa se mantuvo dentro de un amplitud de $13,3^{\circ}$ (figura 5), siendo así esta articulación la que presenta una menor amplitud articular, dentro de las consideradas en este estudio para el miembro anterior.

Galisteo y col (1997) realizaron un estudio comparativo entre dos razas (Dutch Warmblood y Andaluz) al trote, encontrando un rango de movimiento articular para la articulación del hombro de $19,3^{\circ}$ para el Dutch Warmblood (DWB) y de 22,8 para el Andaluz (AN). Cano y col (2001) en un estudio similar, pero utilizando caballos Arabes, Anglo-Arabes y Andaluces, encontraron un rango de movimiento articular de $16^{\circ}$ para el Anglo-Arabe (AA), $25^{\circ}$ para el Arabe (AR) y de $22^{\circ}$ para el Andaluz (AN). Las amplitudes mayores encontradas en estos trabajos pueden deberse a que fueron medidas al trote donde existe una mayor elevación del miembro y con ello se logra una mayor flexión de la articulación 
resultando en una mayor amplitud articular. En el caso de las cuatro razas mencionadas y al igual que en el presente trabajo, la articulación del hombro corresponde a la articulación del miembro anterior que presenta un rango de movimiento articular menor. Al respecto, Holmström y Philipsson (1993) encontraron que caballos con mayor rango de movimiento de hombros están relacionados de manera positiva con movimientos considerados deseables, ya que facilitan el movimiento hacia arriba y adelante.

Para la articulación del codo se describe un ángulo de $150^{\circ}$ en el equino de pie, lográndose amplitudes articulares de $60^{\circ}$ (Kainer 1985); sin embargo para esta articulación no se describe bajo qué condiciones de movimiento se obtuvieron los valores indicados. Para las yeguas Chilotas se obtuvo una máxima extensión de $136,3^{\circ}$ y una máxima flexión de $68,3^{\circ}$ (cuadro 3 ), lográndose un rango de movimiento articular de $67,9^{\circ}$ durante una batida completa al paso guiado a mano (figura 6), siendo así la articulación que presenta un ángulo de flexión mayor en el miembro anterior.

Para la misma articulación, los resultados obtenidos por Galisteo y col (1997), al comparar el DWB con el $\mathrm{AN}$ al trote, dieron un rango de movimiento articular de $57,8^{\circ}$ para el primero y $71,3^{\circ}$ para el segundo, mientras que Cano y col (2001) obtuvieron rangos de movimiento articular al trote de $60,1^{\circ}$ para el AA, 58,4 para el AR y de 67,1 para el AN. Los valores obtenidos al paso para la articulación del codo del Caballo Fino Chilote se asemejan más a los resultados obtenidos al trote para el caballo Andaluz, aunque se debe considerar que se midieron utilizando aires distintos.

En cuanto a la articulación del carpo se obtuvo el valor más alto de extensión dentro de las articulaciones medidas en el miembro anterior $\left(175,4^{\circ}\right.$, cuadro 3$)$ y una máxima flexión de $105,6^{\circ}$ (cuadro 3 ), presentando de la misma manera el mayor rango de movimiento articular para el miembro anterior, a lo largo de una batida completa al paso guiado a mano (figura 7). A pesar de alcanzar valores similares al DWB y al AN para la extensión de la articulación del carpo, las yeguas utilizadas no alcanzaron valores similares de flexión de la articulación, la cual es notoriamente mayor en el AN (Galisteo y col 1997); nuevamente hay que considerar los distintos aires a los que fueron medidos.

Una mayor flexión de las articulaciones del codo y carpo lleva a una mayor elevación del miembro durante una batida y van a dar la característica del aire para la raza. En el caso de la raza AN se encuentran movimientos elevados por una mayor flexión de sus articulaciones, dando lugar a un aire más elegante, mientras que para el DWB prevalecen movimientos más productivos, orientados a alcanzar progresión lineal (Galisteo y col 1997). Algo similar al DWB ocurre con el caballo Arabe, donde al elevar menos el miembro reduce el gasto de energía muscular, la cual es utilizada en progresión y favorece así su uso como raza para competencias de enduro (Cano y col 2001).
Para la articulación de la cadera, se obtuvieron valores de máxima extensión de $81,4^{\circ}$ y de máxima flexión de $63,9^{\circ}$ (cuadro 3 ), con un rango de movimiento articular a través de una batida completa al paso guiado a mano de $17,4^{\circ}$ (figura 8). Esta articulación fue la que presentó un rango de movimiento articular menor en el miembro posterior y además también presentó un rango de movimiento articular inferior a los encontrados en las tres razas descritas al trote por Cano y col (2001).

Para la articulación de la rodilla Kainer (1985) describe un ángulo de $150^{\circ}$ aproximadamente con el caballo de pie. Para las yeguas Chilotas, al paso guiado a mano se obtuvieron valores de máxima extensión de $131,3^{\circ}$ y de máxima flexión de $85,8^{\circ}$ (cuadro 3 ), mientras que se obtuvo un rango de movimiento articular de $45,5^{\circ}$ a través de una batida completa (figura 9). La articulación de la rodilla en conjunto con la cuartilla juega un rol importante en la conformación del miembro en conjunto con el largo de los huesos, ya que entre mayores los ángulos de estas articulaciones en el animal de pie, más rectas van a ser las piernas, y piernas demasiado rectas son reprobables al momento de seleccionar un caballo, ya que no absorben adecuadamente la concusión (Nordby 1971). Este no fue el caso de las 16 yeguas estudiadas en el presente trabajo.

Para la articulación del corvejón, se registraron valores de máxima extensión de $156,9^{\circ}$ y de máxima flexión de $105,9^{\circ}$ (cuadro 3 ), mientras que el rango de movimiento articular que se obtuvo a través de una batida completa fue de 50,9 (figura 10). Kainer (1985) describe un ángulo de $150^{\circ}$ aproximados para la articulación del corvejón con el animal de pie, valor que se asimila al obtenido para la máxima extensión en este estudio.

En general no se encuentran muchas diferencias en las variables angulares para las articulaciones del miembro posterior al comparar diferentes razas, lo cual puede deberse a su función principal de propulsión (Cano y col 2001, Galisteo y col 1999). Las diferencias más significativas se encuentran al comparar variables angulares del miembro anterior, lo cual daría lugar a las particularidades de cada raza para un mismo aire y que lo caracterizan para un determinado uso (Galisteo y col 1997). Esto sería uno de los motivos por los cuales la literatura en general se ha dirigido a la medición de las variables angulares sólo en el miembro anterior. Sería interesante describir las variables angulares del Caballo Fino Chilote al trote para poder llevar a cabo una comparación con otras razas, ya que la mayoría de los trabajos se han realizado utilizando dicho aire.

En relación al ángulo de inclinación pélvica, se obtuvo una máxima inclinación de $23,9^{\circ}$ y una mínima de $15,5^{\circ}$ (cuadro 3 ) con una amplitud a través de una batida de $8,4^{\circ}$ (figura 11). Este rango de movimiento articular es muy similar al encontrado para la raza Anglo-Arabe de $8,3^{\circ}$ por Cano y col (2001). Este movimiento en conjunto con el resto de las variables mencionadas sería el 


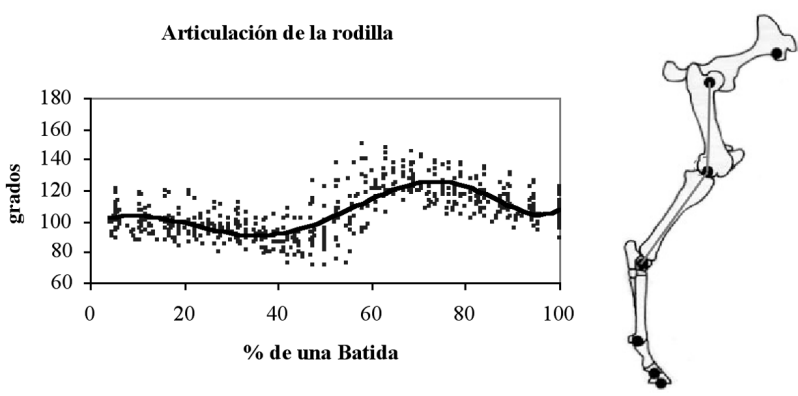

Figura 9. Curva de tendencia de las variaciones angulares (grados) para la articulación de la rodilla de las 16 yeguas, durante una batida completa.

Tendency curve of the angular variables (degrees) at the stifle joint for the 16 mares during a complete stride.

responsable de producir un desplazamiento vertical y lateral del centro de gravedad de $5 \mathrm{~cm}$, durante el cabalgar y el caminar, en el jinete, además de una rotación pélvica de alrededor de $8^{\circ}$ hacia cada lado de la posición central durante la cabalgata (Depauw 1986).

En cuanto al ángulo de inclinación de la escápula, variaciones en éste están relacionadas de manera cercana con la retracción y protracción del miembro, como también con un nivel de performance alto; por otra parte, escápulas muy rectas reciben mayores niveles de concusión y los caballos con este tipo de conformación sufren más de cojeras (Cano y col 2001). Por lo mismo, escápulas que presentan una inclinación apropiada facilitan el libre movimiento de avance de las extremidades anteriores (Nordby 1971). En este caso se encontró un ángulo de inclinación de la escápula de $72,9^{\circ}$ en su máxima extensión y de 53,8 en su máxima flexión (cuadro 3 ), con un rango de movimiento articular durante una batida al paso guiado a mano de $19,2^{\circ}$ (figura 12 ). Valores similares se han encontrado al trote, para las razas AngloArabes $\left(18,7^{\circ}\right)$, Arabes $\left(21,2^{\circ}\right)$ y Andaluces $\left(20,4^{\circ}\right)$ en el estudio realizado por Cano y col. (2001). También

Inclinación Pélvica
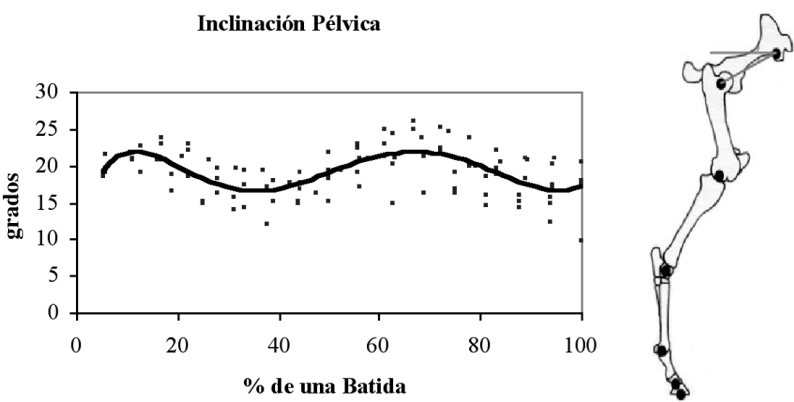

Figura 11. Curva de tendencia de las variaciones angulares (grados) del ángulo de inclinación pélvica para las 16 yeguas, durante una batida completa.

Tendency curve of the angular variables (degrees) for pelvic inclination angle for the 16 mares during a complete stride.

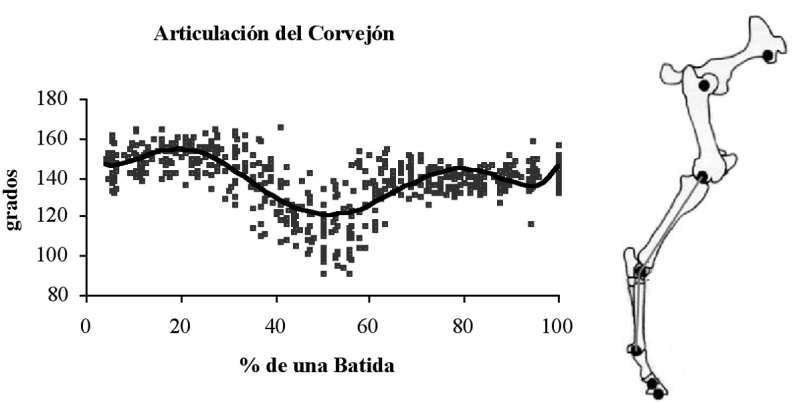

Figura 10. Curva de tendencia de las variaciones angulares (grados) para la articulación del corvejón de las 16 yeguas, durante una batida completa.

Tendency curve of the angular variables (degrees) at the tarsus joint for the 16 mares during a complete stride.

Galisteo y col (1997) obtuvieron valores de $18,5^{\circ}$ para el Dutch Warmblood y de 20, $1^{\circ}$ para el Andaluz, al trote.

En general, el Caballo Fino Chilote presentó características conformacionales y de movimiento que lo harían una raza adecuada para ser utilizada en la actividad de hipoterapia.

Para todos los parámetros angulares medidos en este estudio se encontraron resultados similares a los obtenidos para el Andaluz en los estudios realizados al trote por Cano y col (2001) y por Galisteo y col (1997). El caballo Andaluz ha sido seleccionado tradicionalmente y de manera casi exclusiva por sus características morfológicas y por sus movimientos elegantes y elevados, siendo utilizado mayoritariamente como animal de silla y de trabajo en el campo (Galisteo y col 1997). Esta es una de las razas más antiguas que se conoce y ha jugado un rol importante en el desarrollo de otras razas y por lo tanto sería interesante compararlos, en un estudio futuro, bajo condiciones iguales (aire, velocidad y superficie) con el Caballo Fino Chilote.
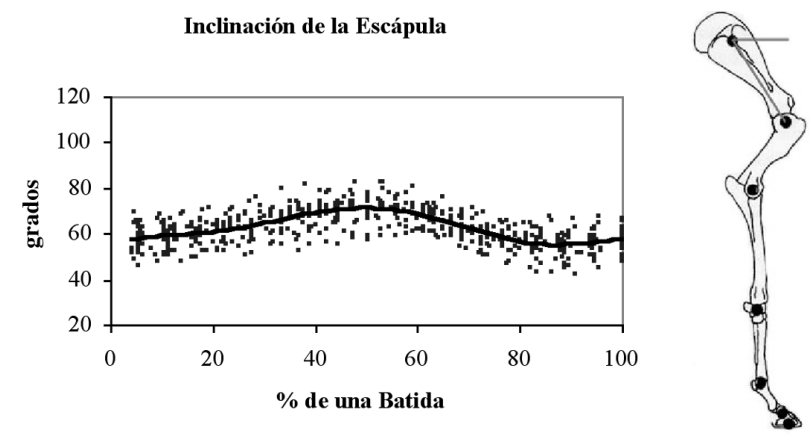

Figura 12. Curva de tendencia de las variaciones angulares (grados) para el ángulo de inclinación de la escápula de las 16 yeguas, durante una batida completa.

Tendency curve of the angular variables (degrees) for scapulae inclination angle for the 16 mares during a complete stride. 


\section{RESUMEN}

Se realizó una descripción de ciertas variables conformacionales y biocinemáticas básicas, al paso guiado a mano, en 16 yeguas de la raza Caballo Fino Chilote.

Las variables conformacionales se midieron en vivo con el uso de huincha métrica y bastón hipométrico. Para obtener las variables biocinemáticas y de tiempo se utilizó un telón, frente al cual se hizo pasar al paso guiado a mano a las 16 yeguas, mientras eran filmadas con una cámara de video Sony Hi-8. El video fue transformado a fotografías con el programa Final Cut, obteniéndose 15 cuadros por segundo en formato JPG, las que se transformaron a formato Tiff para medir las variables angulares de los miembros a través del programa Scion Image. Las variables lineales fueron obtenidas directamente desde el video a través de un televisor Sony pantalla plana. Los datos se analizaron utilizando estadística descriptiva con el programa Microsoft Excel xp.

Los promedios para las variables conformacionales fueron: alzada a la cruz de $117,9 \mathrm{~cm}( \pm 2,6)$, alzada a la espalda 113,3 $\mathrm{cm}( \pm 2,1)$ y alzada a la grupa $119,9 \mathrm{~cm}( \pm 3,2)$. Dentro de las variables lineales, para el largo de batida se obtuvo un promedio de $135,3 \mathrm{~cm}( \pm 8,9)$ y para el largo del paso $69,3 \mathrm{~cm}( \pm 5,8)$. Se midió el tiempo de duración de una batida completa en la cual se obtuvo un promedio de 1,18 segundos, lo cual corresponde a una velocidad promedio de $114,7 \mathrm{~cm} / \mathrm{seg}$. Las variaciones angulares de los miembros se midieron para las articulaciones del hombro, codo y carpo en el miembro anterior y para las articulaciones de la cadera, rodilla y tarso en el miembro posterior; además se obtuvieron valores de inclinación de la escápula y de la pelvis, todos estos a través de una batida completa; se obtuvieron curvas de movimiento para cada articulación durante un ciclo completo además de los rangos articulares.

Además podemos concluir que el Caballo Fino Chilote presenta ciertas características conformacionales y de movimiento que lo hacen apto para el trabajo en hipoterapia.

\section{REFERENCIAS}

Adams OR. 1985. Natural and artificial gaits. Capítulo 13. En Stashak, T. Adam's lameness in horses. $4^{\mathrm{a}}$ edición. Lea y Febiger, Philadelphia, Estados Unidos.

Badoux DM. 1994. Bioestática y biomecánica. Capítulo 5. En Getty R., Sisson, S., Grossman, J. (eds.). Anatomía de los animales domésticos. Tomo 1. $5^{\mathrm{a}}$ edición. Salvat ciencia y cultura. Latinoamérica S.A. de C.V. Iztapalapa D.F., México.

Barrera M. 1998. Parámetros morfológicos y tipificación de polimorfismos antigénicos eritrocitarios y bioquímicos como base del stud book de la raza caballo chilote. Tesis M. V. Universidad Austral de Chile, Facultad de Ciencias Veterinarias, Valdivia, Chile.

Briggs HM. 1969. Razas modernas de animales domésticos. Ed. Acribia, Zaragoza, España.

Buchner HHF. 2001. The advances of clinical biomechanics. Equine vet $J 33$, 430-432.

Cabrera A. 1945. Caballos de América. Editorial Sudamericana. Buenos Aires, Argentina.

Cano MR, J Vivo, F Miró, JL Morales, AM Galisteo. 2001. Kinematic characteristics of Andalusian, Arabian and
Anglo Arabian horses: a comparative study. Res Vet Sci 71, 147-153.

Clayton HM. 1995. Comparison of the stride kinematics of the collected, medium, and extended walks in horses. Am J Vet Res 56, 849-852.

Depauw K. 1986. Horseback riding for individuals with disabilities: Programs, philosophy and research. Adapted physical activity quarterly. 3:217-226.

Dusek F. 1974. Beitrag zum problem der beziehung zwischen der höhe de pferdes und der bewegungsmechanik bei den Hannover-aner-Hengsten. Bayer Landw Jb 52, 209-213.

Ensminger ME. 1973. El caballo en acción. Capítulo 7. En: Ensminger, M.E . 1973. Producción equina. El Ateneo. Buenos Aires, Argentina.

Galisteo AM, J Vivo, MR Cano, JL Morales, F Miró, E Agüera. 1997. Differences between breeds (Dutch Warmblood vs Andalusian Purebred) in forelimbs kinematics. $J$ Equine Sci 8, 43-47.

Galisteo AM, MR Cano, JL Morales, J Vivo, F Miró. 1998. The influence of speed and height at the withers on the kinematics of sound horses at the hand led trot. Vet Res Comm 22, 415-423.

Galisteo AM, J Vivo, F Miró, JL Morales, JG Monterde, MR Cano. 1999. Variaciones en el patrón biocinemático básico del paso de caballos de tres razas guiados de la mano. Arch Zoot 48, 327-335.

Holmström M, L Magnusson, J Philipsson. 1990. Variation in conformation of Swedish Warmblood horses and conformational characteristics of elite sport horses. Equine vet $J$ 22, 186-193.

Holmström M, Philipsson J. 1993. Relationships between conformation, performance and health in 4 year old Swedish Warmblood riding horses. Livestock Prod Sci 33, 293-312.

Kainer R. 1985. Functional anatomy of equine locomotor organs. Capítulo 1. En Stashak, T. 1985. Adam's lameness in horses. $4^{\mathrm{a}}$ edición. Lea y Febiger. Philadelphia, Estados Unidos.

Lehmann J. 1999. Análisis de la marcha, diagnóstico y manejo. Capítulo 4. En: Krusen. 1999. Medicina física y rehabilitación. $4^{a}$ edición. Editorial médica panamericana. Madrid, España.

Morales JL, M Machado, J Vivo, AM Galisteo, E Agüera, F Miró. 1998. Angular kinematic patterns of limbs in elite and riding horses at trot. Equine vet J 30, 528-533.

Nordby J. 1971. Caballos: Selección, preparación y exposición. Editorial Albatros. Buenos Aires, Argentina.

Peham C, T Licka, D Girfler, M Scheidi. 2001. The influence of lameness on equine stride length consistency. The Veterinary journal 162, 153-157.

Pinochet JL. 1980. Estudio hipométrico y morfológico del caballo de raza criolla chilena y su posible cambio tipológico. Tesis, M. V. Universidad de Chile, Facultad de Ciencias Veterinarias y Pecuarias, Santiago, Chile.

Soto F. 2002. La hipoterapia: Caballos que curan. II Congreso nacional de nuevas tecnologías y necesidades educativas especiales, 19-21 septiembre, 2002. Murcia, España.

Voeltz J. 1996. Descripción morfológica básica del caballo chilote y su distribución en la Isla de Chiloé. Tesis M. V. Universidad Austral de Chile, Facultad de Ciencias Veterinarias, Valdivia, Chile. 